\title{
Splicing factor mutations in MDS RARS and MDS/MPN-RS-T
}

\author{
Akihide Yoshimi ${ }^{1} \cdot$ Omar Abdel-Wahab $^{1,2}$
}

Received: 27 March 2017 / Revised: 20 April 2017 / Accepted: 23 April 2017 / Published online: 2 May 2017

(C) The Japanese Society of Hematology 2017

\begin{abstract}
Spliceosomal mutations, especially mutations in $S F 3 B 1$, are frequently (>80\%) identified in patients with refractory anemia with ringed sideroblasts (RARS) and myelodysplastic/myeloproliferative neoplasms with ringed sideroblasts and thrombocytosis (MDS/MPN-RS-T; previously known as RARS-T), and $S F 3 B 1$ mutations have a high positive predictive value for disease phenotype with ringed sideroblasts. These observations suggest that $S F 3 B 1$ mutations play important roles in the pathogenesis of these disorders and formation of ringed sideroblasts. Here we will review recent insights into the molecular mechanisms of mis-splicing caused by mutant $S F 3 B 1$ and the pathogenesis of RSs in the context of congenital sideroblastic anemia as well as RARS with SF3B1 mutations. We will also discuss therapy of $S F 3 B 1$ mutant MDS, including novel approaches.
\end{abstract}

Keywords $S F 3 B 1 \cdot$ Ringed sideroblast · Splicing · RARS · MDS

Omar Abdel-Wahab

abdelwao@mskcc.org

1 Human Oncology and Pathogenesis Program, Memorial Sloan Kettering Cancer Center, Weill Cornell Medical College, Zuckerman 601, 408 East 69th Street, New York, NY 10065, USA

2 Leukemia Service, Department of Medicine, Memorial Sloan Kettering Cancer Center, New York, NY 10065, USA

\section{Background}

\section{Disease overview of RARS and MDS/MPN-RS-T (RARS-T)}

Ringed sideroblasts (RS) are erythroid precursors containing iron-loaded mitochondria surrounding more than a third of nuclear circumference, which can be detected by Prussian blue staining. The recent World Health Organization (WHO) classification of myeloid neoplasms (WHO 2016 [1]) categorized the clonal diseases with RS within myelodysplastic syndromes (MDS) as refractory anemia with ringed sideroblasts (RARS), refractory cytopenias with multilineage dysplasia and ringed sideroblasts (RCMDRS), or myelodysplastic/myeloproliferative neoplasms with RS and thrombocytosis (MDS/MPN-RS-T; previously known as RARS with thrombocytosis (RARS-T) in the WHO 2008 classification [2]). In some cases, RS is also detected in patients with other types of MDS [refractory anemia with excess blasts $1 / 2$ (RAEB-1/2) and unclassifiable MDS (MDS-U)], MPN [essential thrombocythemia (ET) and primary myelofibrosis (PMF)], and MDS/MPN overlap syndromes (chronic myelomonocytic leukemia and unclassified MDS/MPN). In addition, there are non-clonal conditions also associated with RS. These include acquired conditions such as exposures to excess alcohol, lead, zinc, isoniazid, chloramphenicol, linezolid, and penicillamine, as well as inherited conditions, where a mutation in one of the following three mitochondrial functions/pathways has been know to cause sideroblastic anemia (SA): (i) heme synthesis, (ii) iron-sulfur $(\mathrm{Fe}-\mathrm{S})$ cluster biogenesis and protein synthesis, or (iii) mitochondrial respiration (Table 1). Historically, our understanding of the pathogenesis of SAs has been greatly facilitated by discovering the genetic defects 
Table 1 Causes of bone marrow ringed sideroblasts

(1) Clonal disease

1. Myelodysplastic syndromes (MDS)

(i) Refractory anemia with ringed sideroblasts (RARS)

(ii) Refractory cytopenias with multilineage dysplasia and ringed sideroblasts (RCMD-RS)

(iii) Refractory anemia with excess blasts-1/2 (RAEB-1/2)

(iv) Unclassifiable MDS (MDS-U)

2. Myeloproliferative neoplasms (MPN)

(i) Essential thrombocythemia (ET)

(ii) Primary myelofibrosis (PMF)

3. MDS/MPN overlap syndromes

(i) MDS/MPN with ringed sideroblasts and thrombocytosis (MDS/MPN-RS-T) (RARS-T)

(ii) Chronic myelomonocytic leukemia (CMML)

(iii) Unclassified MDS/MPN

(2) Non-clonal conditions

1. Hereditary sideroblastic anemia

(i) Defects in heme synthesis

XLSA (X-linked sideroblastic anemia) - mutations in ALAS2 and SLC25A38

(ii) Defects in Fe-S cluster biogenesis and protein synthesis

XLSA/A (X-linked sideroblastic anemia with ataxia)—mutations in ABCB7 and GLRX5

(iii) Defects in mitochondrial respiration

MLASA (mitochondrial myopathy, lactic acidosis, and SA) - mutations in PUS1 and YARS2

PMPS (Pearson's marrow-pancreas syndrome)—deletions, rearrangements, or duplications of mitochondrial DNA

SIFD (congenital SA associated with B-cell immunodeficiency, periodic fevers, and developmental delay)—mutations in TRNTI

NDUFB11-mutated sideroblastic anemia

HSPA9-mutated sideroblastic anemia

2. Acquired conditions

(i) Excess alcohol

(ii) Drug exposure (isoniazid, chloramphenicol, linezolid, and penicillamine)

(iii) Lead, zinc toxicity

(iv) Copper deficiency

that affect these mitochondrial functions. Knowledge learned from understanding SAs may also help elucidate the pathogenesis of clonal diseases with RS due to recurrent genetic alterations found in these diseases (described below).

Clinically, RARS is a low-risk form of MDS characterized by anemia, dysplasia in the erythroid lineage, and the presence of $\geq 15 \%$ RS in bone marrow (BM) erythroid progenitors. Dysplasia should be minimal in non-erythroid lineages and BM myeloblasts should be less than $<5 \%$. RARS accounts for 3-15\% of all MDS. The form of MDS known as Refractory Cytopenias with Multilineage Dysplasia (RCMD) should be considered in the differential diagnosis of RARS. In contrast to RARS, RCMD-RS shows bicytopenia or pancytopenia, and dysplasia affecting $>10 \%$ of the cells in multiple lineages. Generally, patients with RCMD have worse outcome than patients with RARS [3].

RARS-T was previously classified as a provisional entity with overlapping features of MDS and MPN in WHO 2008 [2]. However, recent genetic analysis of this disease entity has uncovered highly frequent mutations in the spliceosomal gene $S F 3 B 1$, providing support that RARS-T is a distinct entity, and RARS-T was, therefore, named as MDS/ MPN-RS-T and considered as a distinct entity in WHO 2016 [1]. MDS/MPN-RS-T is diagnosed when there is thrombocytosis $\left(\geq 450 \times 10^{9} / \mathrm{L}\right)$ associated with refractory anemia, dyserythropoiesis in the BM with RS accounting for $\geq 15 \%$ of erythroid precursors, and megakaryocytes with features resembling those in essential thrombocytosis (ET) or primary myelofibrosis (PMF).

\section{Genetic profiles of RARS and MDS/MPN-RS-T and their impact on outcome}

In 2011, recurrent somatic mutations in genes encoding spliceosome components, including SF3B1, serine and arginine-rich splicing factor 2 (SRSF2), U2 small nuclear RNA auxiliary factor $1(U 2 A F 1)$, and zinc finger CCCH-type RNA binding motif and serine/arginine rich 2 (ZRSR2), were first described in patients with MDS [4, 5]. Amongst these, $S F 3 B 1$ mutations are most common in patients with RARS, accounting for $\sim 80 \%$ of cases, whereas mutations of other spliceosome components are relatively rare (SRSF2 5.5\%; U2AF1 0\% [5]). Nearly all $S F 3 B 1$ mutations are located within the Huntingtin, elongation factor 3, protein phosphatase 2A, and the yeast PI3kinase TOR1 (HEAT) domains of SF3B1 and occur as heterozygous substitutions. A single mutation (SF3B1K700E) accounts for $>50 \%$ of $S F 3 B 1$ mutations in MDS, followed by mutations at the residue K666. Clonal analysis based on the variant allele frequency of $S F 3 B 1$ and other co-existing mutations revealed that mutations in $S F 3 B 1$ are present in the dominant clone in most cases. Interestingly, the percentage of BM RS is highly correlated with $S F 3 B 1$ mutant allele burden [4, 6-8], which strongly suggests that mutant 
SF3B1 directly or indirectly contributes to the formation of RS. In fact, SF3B1 mutations have a high positive predictive value for disease phenotype with RS of $97.7 \%$, whereas the absence of these mutations has an equivalent negative prediction value [7]. These data make $S F 3 B 1$ the first gene to be strongly related to a specific morphology in myeloid disorders.

In contrast to the diagnostic utility of SF3B1 mutations in RS, the prognostic impact of SF3B1 mutations in MDS with RS is controversial. For example, Malcovati et al. studied the influence of $S F 3 B 1$ mutations on outcome in 293 patients with myeloid neoplasms with $1 \%$ or more RS [6]. In this cohort, $S F 3 B 1$ mutations were detected in 129 of 159 patients $(81 \%)$ with RARS or RCMD-RS, and multivariate analysis demonstrated that patients with $S F 3 B 1$ mutations had significantly better overall survival [hazard ratio (HR) 0.37] and lower cumulative incidence of disease progression (HR 0.31). However, SF3B1 mutations in a similar-sized cohort [288 patients with low-risk MDS; MD Anderson lower risk prognostic scoring system (LRPSS)] did not impact overall survival (OS) or leukemia-free survival (LFS) [9]. In this study, SF3B1 mutations were detected in $78 \%$ of patients with BM RS and frequently co-occurred with DNA methyltransferase 3A (DNMT3A) mutations $(p<0.001)$. Patnaik et al. studied 107 patients with MDS-RS, including 48 with RARS and 43 with RCMD-RS. SF3B1 mutations were identified in 35 RARS (73\%) and 16 RCMD-RS (37\%), respectively, and the presence of SF3B1 mutations was associated with better OS $(p<0.01)$ and LFS $(p<0.01)$ in univariate analysis [10]. However, mutant $S F 3 B 1$ was not identified as an independent prognostic factor in further analysis in this study.

Mutational profiles of MDS/MPN-RS-T (RARS-T) have also been studied in several large cohorts [11-13]. According to these studies, commonly mutated genes in MDS/MPN-RS-T include SF3B1 (85-90\%), JAK2V617F (33-77\%), TET2 (10-25\%), DNMT3A (13-15\%), and ASXL1 (15-29\%). Approximately 50\% of MDS/MPN-RST patients have both $S F 3 B 1$ and $J A K 2$ mutations, providing an intriguing genetic explanation for the hybrid nature of MDS/MPN-RS-T as being between an MDS and an MPN. This unique genetic profile is particularly valuable for diagnosis, as it is sometimes difficult to distinguish MDS/MPNRS-T from RARS with reactive thrombocytosis based on morphology alone. Although mutations in the calreticulin gene $(C A L R)$ have been reported to be mutually exclusive with $J A K 2$ and $M P L$ mutations and are detected in $67-71 \%$ of ET and 56-88\% of PMF [14, 15] with wild-type (WT) $J A K 2$ or $M P L$, mutations in CALR $(0-3 \%)$ and $M P L$ (1-3\%) are not common in MDS/MPN-RS-T [14-16].

According to a large study performed by Broseus et al. [17], patients with MDS/MPN-RS-T have a better median OS than RARS (76 versus 63 months) but an inferior OS when compared to patients with ET (117 months). The leukemic transformation rates are similar in MDS/MPN-RS$\mathrm{T}(1.8 / 100$ years) and RARS (2.4/100 years) and higher in MDS/MPN-RS-T in comparison to ET (0.7/100 years). In contrast to RARS, SF3B1 mutations do not seem to have any prognostic impact in MDS/MPN-RS-T. On the other hand, presence of anemia $(p=0.02)$, abnormal karyotype $(p=0.04)$, SETBPI mutations $(p=0.04)$, and ASXL1 mutations $(p=0.08)$ have recently been identified as independent predictors of inferior survival in multivariate analysis of 82 patients with MDS/MPN-RS-T [13]. Based on these observations, an HR-weighted prognostic model was developed, in which patients receive 2 points for an abnormal karyotype, 1 point for either SETBP1 and/or ASXL1 mutations, and 1 point for hemoglobin level $<10 \mathrm{~g} / \mathrm{dL}$. Risk stratification categorized patients into three categories: low ( 0 point), intermediate ( 1 point), and high ( 2 or more points), with median OS of 80,42 , and 11 months, respectively [13].

\section{Pathophysiology of ring sideroblasts}

In contrast to the common pathological features of RS, the underlying molecular mechanisms leading to mitochondrial iron accumulation in SAs are greatly heterogeneous. As described above, our understanding of the pathogenesis of SAs has emerged from discovery of the genetic abnormalities underlying various congenital SAs, and these genetic events effect three processes: (i) heme synthesis, (ii) $\mathrm{Fe}-\mathrm{S}$ cluster biogenesis and protein synthesis, and (iii) mitochondrial respiration (reviewed recently $[18,19])$ (Table 1). Amongst these congenital diseases, X-linked sideroblastic anemia (XLSA) is the most common SA, accounting for $40 \%$ of cases [20]. The most frequent form of XLSA is caused by defects involving $\delta$-aminolevulinate synthase (5-aminolevulinate synthase $2 ; A L A S 2)$. ALAS2 is located at Xp11.21 and encodes the first and rate-controlling enzyme of the heme biosynthesis pathway that mediates the condensation of glycine and succinyl-CoA to form 5-aminolevulic acid (ALA) in erythroid cells [21, 22]. More than 80 distinct ALAS2 mutations have been identified in $>120$ unrelated probands. Most of ALAS2 mutations are heterozygous missense mutations that lead to loss-offunction via decrease in ALAS2 expression or defects in catalysis, substrate, or cofactor affinity. Following the discovery of ALAS2 mutations, mutations in solute carrier family 25 member 38 (SLC25A38), the second causal gene implicated in SAs, were reported in 2009 [23]. SLC25A38 encodes an erythroid-specific mitochondrial inner membrane carrier protein. More than 30 patients with SLC25A38-mutated XLSA have been reported so 
far, accounting for $\sim 15 \%$ of congenital SAs, and mutations in SLC25A38 include missense as well as nonsense and splicing errors [23, 24]. SLC25A38 is thought to be an amino acid transporter required for importing glycine across the inner mitochondrial membrane in the initial steps of heme synthesis. Although transmembrane protein 14C (TMEM14C) is also involved in the terminal steps of heme synthesis pathway [25], no mutations in this gene have been reported in congenital SAs so far.

Two subsequently recognized forms of SA, XLSA with ataxia (XLSA/A) and glutaredoxin 5 (GLRX5) deficiencies, are due to mutations in genes involved in the $\mathrm{Fe}-\mathrm{S}$ pathway, an essential pathway of mitochondrial iron utilization. $\mathrm{Fe}-\mathrm{S}$ clusters are generated from iron imported into mitochondria and participate in proteins regulating heme synthesis, cellular iron uptake, and iron storage, such as ferrochelatase $(\mathrm{FECH}$; the final enzyme in the heme biosynthesis pathway) and iron regulatory protein 1 (IRP1). XLSA/A has been described in four families so far and is characterized by mild to moderate microcytic SA accompanied by neurologic deficits of delayed motor and cognitive development, impaired coordination, and cerebellar hypoplasia $[26,27]$. XLSA/A is caused by missense mutations in ATP-binding cassette sub-family $\mathrm{B}$ member $(A B C B 7)$ [28]. This gene is located at Xp13.3 and encodes an essential component of the $\mathrm{Fe}-\mathrm{S}$ cluster machinery, which is postulated to participate in the export of $\mathrm{Fe}-\mathrm{S}$ clusters generated in mitochondria [29]. It is, therefore, speculated that iron remains trapped in mitochondria and the level of $\mathrm{Fe}-\mathrm{S}$ cluster-dependent enzyme activity is decreased in $A B C B 7$ mutant cells. In fact, it has been shown that expression of mutant $A B C B 7$ impairs the maturation of cytosolic $\mathrm{Fe}-\mathrm{S}$ proteins in yeast in which $A T M 1$, a functional orthologue of $A B C B 7$, is deleted, whereas expression of wild-type (WT) $A B C B 7$ fully rescued the defect [28]. Importantly, defects in $\mathrm{ABCB} 7$ are predicted to decrease the activity of $\mathrm{FECH}$ via impaired binding between these two proteins, thus blocking the heme biosynthesis. In 2007, a patient with microcytic hypochromic SA presenting in midlife was reported to have a homozygous splice site mutation in GLRX5 [30], which resulted in reduced mRNA and protein levels of GLRX5. GLRX5 is a mitochondrial protein involved in $\mathrm{Fe}-\mathrm{S}$ cluster biogenesis. Mutations in GLRX5 result in loss of $\mathrm{Fe}-\mathrm{S}$ cluster in IRP1, which in turn suppresses ALAS2 translation by binding to the iron responsive element positioned at the 5'-untranslated region of ALAS2. In addition, a global defect in mitochondrial protein translation can lead to congenital SAs, although the mechanism for iron accumulation in mitochondria is unclear.

Congenital SAs caused by genetic abnormalities classified into the third category include mitochondrial myopathy, lactic acidosis, and SA (MLASA) and Pearson's marrow-pancreas syndrome (PMPS). MLASA is an autosomal recessive disorder with muscle weakness, normocytic anemia, and lactic acidosis. Gene mutations in pseudouridine synthesis 1 (PUS1) and mitochondrial tyrosyl transfer RNA synthetase 2 (YARS2) result in MLASA [31, 32]. Mutations in these genes are likely to cause defects in decreased mitochondrial protein synthesis and respiratory chain dysfunction. Deletions, rearrangements, or duplications of mitochondrial DNA also induce the rare syndromic disorder with SA, known as PMPS, which is accompanied with metabolic acidosis and endocrine pancreatic insufficiency [33]. Recently, mutations in NADH:ubiquinone oxidoreductase subunit B11 (NDUFB11) [34], HSPA9 [the mitochondrial heat-shock protein family A (Hsp70) member 9] [35], and TRNT1 (tRNA nucleotidyl transferase 1) in SIFD (congenital SA associated with B-cell immunodeficiency, periodic fevers, and developmental delay) [36] have been identified to cause SAs in this category as well.

Given that RARS and other clonal myeloid diseases with RS have a similar morphological phenotype as SAs with regard to iron overload in mitochondria, discoveries from congenital SAs may provide us with insights into the pathogenesis of SAs observed in clonal conditions. In fact, gene expression analysis of RARS has identified dysregulated genes involved in iron metabolism and mitochondrial functions relative to refractory cytopenias with unilineage dysplasia (RCUD) and control samples [37]. For example, expression of mitochondrial transporters (SLC25A37 and $S L C 25 A 38$ ) and aminolevulinate dehydratase (ALAD), which has a direct role in heme formation, are deregulated in RARS. Moreover, significant differences are observed between patients with $S F 3 B 1$ mutations and patients without SF3B1 mutations [37]. Further investigations on these deregulated genes in relation to mis-splicing mediated by mutant SF3B1 are reviewed below.

\section{General mechanisms of pre-mRNA splicing and mis-splicing by mutant SF3B1}

As noted earlier, SF3B1 mutations, but not mutations in other spliceosomal genes, are clearly correlated with RS in MDS. Given the essential role of SF3B1 in precursor messenger RNA (pre-mRNA) splicing, this observation strongly supports the idea that $S F 3 B 1$ mutations play fundamental roles in the disease formation of RARS and other myeloid disorders with RS via pre-mRNA missplicing. Pre-mRNA splicing is the processes of removing introns from pre-mRNA, representing a critical step in the post-transcriptional regulation of gene expression. Splicing is carried out in the nucleus by an enzymatic complex known as the spliceosome. The spliceosome consists of 5 small nuclear ribonucleoproteins (snRNPs; U1, U2, U4, U5, and U6 snRNPs), each of which contains its own 
small nuclear RNA (snRNA), and more than 200 related proteins. Splicing catalysis involves two sequential transesterification reactions that are achieved in a complex multistep process (reviewed in Refs. [38-43]), where base pairing of snRNAs to conserved sequences on pre-mRNA as well as interactions of a variety of splicing accessory proteins and RNA-protein interactions are essential in guiding this dynamic process. An intron is defined by four consensus elements: (i) the $5^{\prime}$ splice site $\left(5^{\prime} \mathrm{SS}\right.$; located at the $5^{\prime}$ end of the intron), (ii) the $3^{\prime} \mathrm{SS}$ (the $3^{\prime}$ end of the intron where the AG dinucleotide is located at the intronexon junction), (iii) the branch point sequence (BPS) (located upstream of the $3^{\prime} \mathrm{SS}$ ), and (iv) the polypyrimidine tract (located between the BPS and the $3^{\prime} \mathrm{SS}$ ) (Fig. 1a). U1 snRNA, U2AF1, splicing factor 1 (SF1), and U2AF2 bind the sequences noted above to form complex E. This complex augments the recruitment of U2 snRNP to the BPS. SF3B1 is a component of U2 snRNP and is involved in the binding to the BPS. Following binding of U2 snRNP, other complexes join to induce conformational rearrangements and the subsequent two-step esterification reactions to mediate excision of the intron and ligation of the proximal and distal exon to generate mature mRNA (Fig. 1a).

\section{Pathological significance of SF3B1 mutations in RARS and RARS-T}

Since the initial discovery of spliceosomal mutations (reviewed in [44]), major advances have been made in understanding the biological consequences and molecular mechanisms of spliceosomal mutations. Our understanding of the roles of mutant $S F 3 B 1$ has been greatly promoted through analysis of the phenotypes of genetically engineered mice and molecular analysis of

\section{A}

\section{Splicing in SF3B1 WT cells}

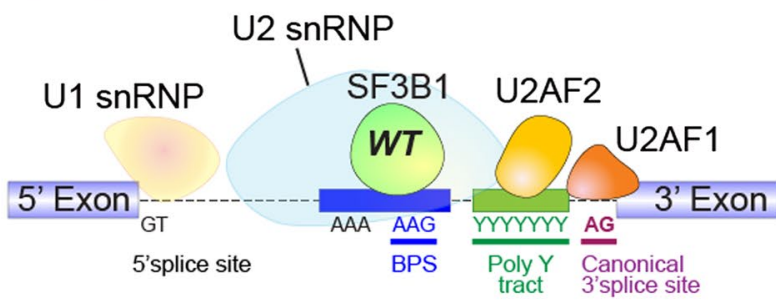

B

Mis-splicing in SF3B1 mutant cells
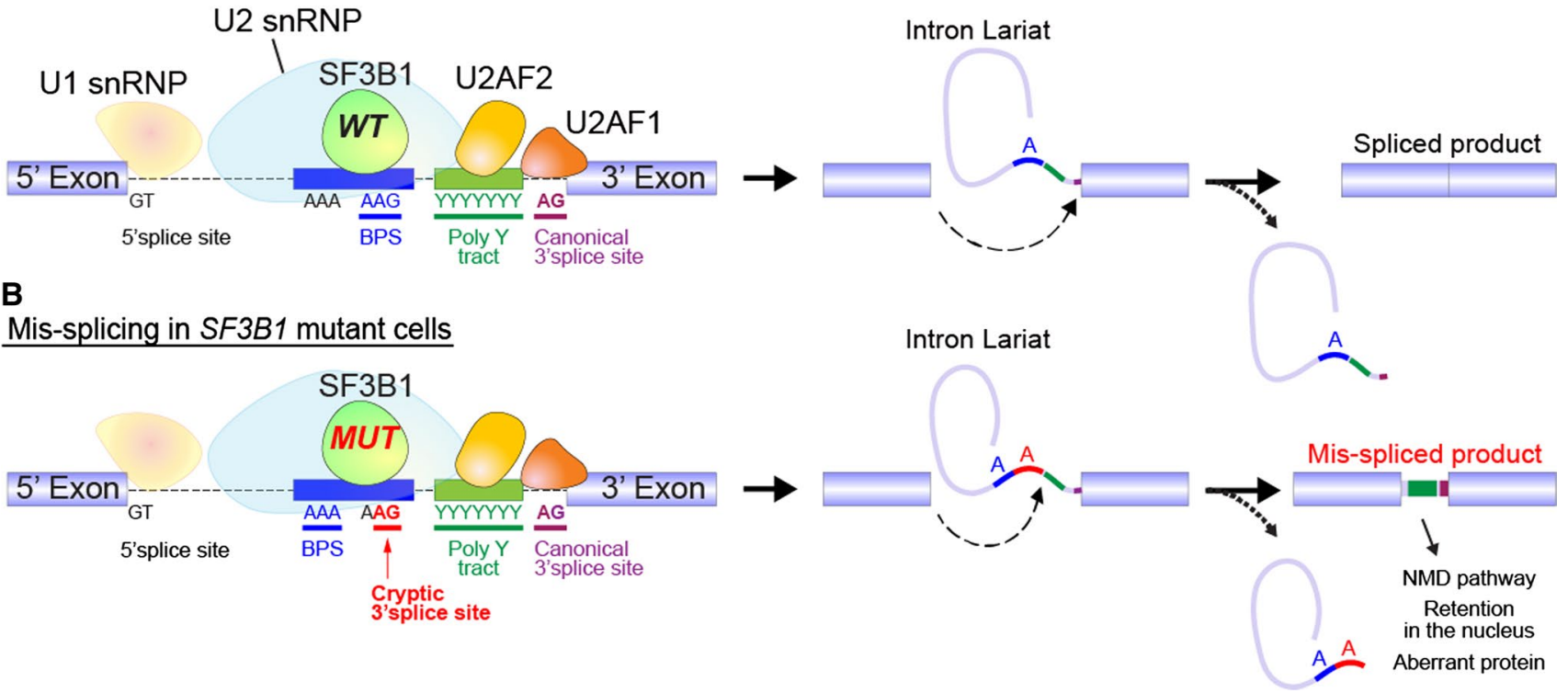

Fig. 1 Splicing catalysis, the spliceosome assembly pathway, and mechanisms of splice site selection in SF3B1 wild-type (WT) and mutant (MUT) cells. a Diagram of the 2 sequential transesterification reactions that represent the crucial catalytic steps in intron removal during splicing. An adenine nucleotide (termed the "invariant adenine") of the branch point sequence (BPS) initiates the first transesterification and generates a free $5^{\prime}$ exon and an intron- $3^{\prime}$ exon lariat. The $3^{\prime}$ end hydroxyl of the free $5^{\prime}$ exon then attacks the intron- $3^{\prime}$ exon junction, completing the splice and releasing a lariat RNA intron. a Mechanisms of splice site selection in SF3B1 WT cells. An intron is defined via (i) the $5^{\prime}$ splice site, (ii) the $3^{\prime}$ splice site, (iii) the branch point sequence (BPS), and (iv) the polypyrimidine (Poly$\mathrm{Y})$ tract. The definition of an intron depends on recognition of the $5^{\prime}$ splice site and BPS by U1 and U2 small nuclear ribonucleoproteins (snRNPs), respectively. The earliest complex (complex E) is established by binding of (i) U1 snRNP to the $5^{\prime}$ splice site, (ii) splicing factor 1 (SF1) to the BPS, (iii) U2AF2 (also known as U2AF65) to the polypyrimidine tract, and (iv) U2AF1 (also known as U2AF35) to the $3^{\prime}$ SS. Formation of complex E in turn enhances the recruitment of U2 snRNP to the BPS and leads to the formation of complex A. SF3B1, a component of U2 snRNP, is involved in the binding to the BPS. Further assembly of other spliceosomal components, conformational rearrangements, and the two-step esterification reactions that mediate excision of the intron and ligation of the proximal and distal exon to synthesize mature mRNA follows these reactions. b Potential mechanisms of splice site selection in SF3B1 mutant cells. Our current understanding suggests that aberrant splicing mediated by mutant SF3B1 utilizes a cryptic AG, and requires the canonical $3^{\prime}$ splice site. Moreover, the cryptic AG requires the downstream canonical polypyrimidine tract to cause alternative splicing, and three adenosines upstream of the cryptic AG are part of the BPS for mutant SF3B1 but not for WT SF3B1 
isogeneic cell lines, yeast models, mouse models, and patient samples.

Two recent studies addressed the biological effects of expression of $S F 3 B 1 \mathrm{~K} 700 \mathrm{E}$ in hematopoiesis in vivo through generation of knock-in (KI) mice with Sf3b1K700E expression as well as RNA sequence analysis of both these mice and MDS patient samples [45, 46]. In both models, $M x 1$-Cre $S f 3 b 1^{+/ K 700 E}$ conditional knock-in mice develop progressive macrocytic anemia with impaired terminal erythroid differentiation. Although these KI mice also have increased CD $150^{+} \mathrm{CD} 48^{-}$LSK $_{\text {(lineage }}{ }^{-} \mathrm{Sca}^{+}$ $\mathrm{cKit}^{+}$) long-term repopulating hematopoietic stem cells (LT-HSCs), expression of $S f 3 b 1^{\mathrm{K} 700 \mathrm{E}}$ was associated with impaired reconstitution capacity compared to WT control mice. Based on the clinical observation that SF3BI and TET2 mutations commonly co-occur in patients with MDS [47, 48], Obeng et al. further crossed $S f 3 b 1^{+/ \mathrm{K} 700 \mathrm{E}}$ mice with conditional Tet 2 knockout $\left(T e t 2^{-/-} \mathrm{KO}\right)$ mice. Tet 2 loss resulted in exacerbation of the macrocytic anemia and impaired terminal erythroid differentiation caused by $S f 3 b 1 \mathrm{~K} 700 \mathrm{E}$ expression and rescued the impaired competitive repopulating capacity of $S f 3 b 1$ mutant cells, suggesting that phenotypes of double-mutant mice recapitulate fundamental characteristics of MDS. In this latter study, RNA-seq of LK (lineage ${ }^{-} \mathrm{cKit}^{+}$) myeloid progenitors identified 72 aberrant splicing events that were specific to $S f 3 b 1$ K700E-expressing cells, with 48 of 72 (67\%) of these events representing alternative $3^{\prime}$ splicing events. Extensive RNA-seq analysis of six SF3B1 mutants and four $S F 3 B 1$ WT MDS patient samples also showed that the most common aberrant splicing event was usage of alternative $3^{\prime}$ splice site $(68 \%)$. The location of the cryptic $3^{\prime}$ splice site in mouse and human cells was both between -15 and -24 nucleotides upstream of the canonical $3^{\prime}$ splice site (Fig. 1b), which is similar to RNA-seq data from lineage ${ }^{-} \mathrm{BM}$ cells of $S f 3 b 1^{+/ \mathrm{K} 700 \mathrm{E}}$ mice generated by Mupo et al. [46], isogenic cell lines [49], or clinical samples from patients with a variety of cancer types with $S F 3 B 1$ mutations $[49,50]$.

Darman et al. further studied the motif sequences associated with cryptic and canonical AG dinucleotides in SF3B1 mutant cells and found that (i) pyrimidines are enriched upstream of the canonical AG, which is in agreement with the presence of the polypyrimidine tract, (ii) the cryptic AGs are associated with a short and weak polypyrimidine tract, and (iii) adenosines are enriched upstream of the cryptic AG in both mouse and human cells expressing mutant SF3BI (Fig. 1b) [49]. The finding that adenosines are enriched upstream of the cryptic AG is particularly interesting because these adenosines could play an important role when mutant SF3B1 recognizes a different BPS. To understand the roles of $3^{\prime}$ SS sequences in early assembly of the spliceosome, particularly how the U2AF2/U2AF1 complex recognizes the polypyrimidine tract and AG, they selected the exon 9/exon 10 junction in ZDHHC16 to develop a series of minigenes because this event is robustly mis-spliced by all SF3B1 hotspot mutants. These experiments demonstrated the following insights: (i) the cryptic AG is necessary for $S F 3 B 1$-mutant aberrant splicing, (ii) the short polypyrimidine tract upstream of the cryptic AG is not recognized by the U2AF complex, (iii) mutant SF3B1 requires the canonical $3^{\prime} \mathrm{SS}$, (iv) the cryptic AG requires the downstream canonical polypyrimidine tract to cause alternative splicing, ( $v$ ) three adenosines between -33 and -35 nucleotides upstream of canonical AG are part of the BPS for mutant SF3B1 but not for WT SF3B1, and (vi) -30 and -34 adenosines are the BPs used for WT and mutant SF3B1, respectively. These observations provide at least one model to explain the mechanisms of differential splicing caused by WT and mutant SF3B1 (Fig. 1b).

The regions of SF3B1 affected by mutations in cancer are remarkably conserved amongst species and this has allowed two groups to generate mutations in the yeast orthologue of SF3B1, termed Hsh155 [51, 52]. In both of these studies, MDS disease-associated mutations in SF3B1 altered interaction between SF3B1 and the protein Prp5, which is a helicase responsible for the first ATP-dependent step in splicing. Based on these results, further work is now needed to demonstrate how altered interaction between Hsh 155 and Prp5 contribute to aberrant splicing by SF3B1 and highlight the potential for further aberrant protein-protein interactions generated by disease-associated mutations in SF3B1.

While we still do not fully understand how aberrant splicing mediated by mutant SF3B1 relates to the phenotype of RS, it has been shown that mRNA and protein expression levels of ABCB7 are downregulated in B-lymphoid leukemia (Nalm-6) cells expressing SF3B1K700E compared to isogeneic $S F 3 B 1$ WT Nalm-6 cells due to aberrant splicing of $A B C B 7$ leading to NMD [49]. These results may at least partially explain the association between downregulation of $A B C B 7$ in RARS and the presence of $S F 3 B 1$ mutations; however, further demonstration of this event with mutations in $S F 3 B 1$ other than K700E is currently lacking. Moreover, it will be important to evaluate the effect of restoration of $A B C B 7$ aberrant splicing and expression in the setting of continued expression of $S F 3 B 1 \mathrm{~K} 700 \mathrm{E}$ to fully confirm the importance of this single splicing event to RS pathogenesis.

Recurrent missense mutations in SF3B1 are generally considered as change-of-function mutations. The vast majority of SF3B1 mutations identified in MDS or other solid tumors are clustered in HEAT domains, mainly involving a few hot spot amino acid position. Recent 
structural insights into the SF3B1 structure have elucidated that recurrent mutations in SF3B1 occur at sites critical for contacting the BPS [53]. In addition, two studies of $S f 3 b l$ KO mice demonstrated that haploinsufficiency of $S f 3 b l$ leads to reduction of hematopoietic stem and progenitor cells (HSPCs) and compromised reconstitution capacity in competitive and non-competitive transplantation [54, 55], similar to the phenotypes of $S f 3 b 1 \mathrm{KI}$ mice [45]. However, RNA-seq analysis of LSK cells from $S f 3 b 1$ heterozygous KO mice suggests that heterozygous loss of $S f 3 \mathrm{bl}$ does not confer the same changes in splicing as the point mutations in SF3B1 described in RS [54]. In aggregate, these observations indicate that $S F 3 B 1$ mutations confer a change-of-function.

The other issue to be discussed regarding the phenotype of $S f 3 b 1 \mathrm{KI}$ and KO mice is the impact of the mutant allele upon the formation of RS. There are conflicting reports regarding whether $S f 3 b 1$ haploinsufficiency causes RS formation. Visconte et al. described rare sideroblasts in the BM of $S f 3 b 1^{+/-}$mice $[56,57]$. In contrast, two other groups quantified the number of RSs in the BM of the same $S f 3 b 1^{+/-}$mice [58] and $S f 3 b 1^{+/+}$mice and found no differences between these animals $[54,55]$. Furthermore, both independent strains of conditional $S f 3 b 1^{+/ \mathrm{K} 700 \mathrm{E}}$ mice $[45$, 46] did not show any significant increase in RSs in BM or peripheral blood, suggesting that physiologic expression of $S f 3 b 1 \mathrm{~K} 700 \mathrm{E}$ does not cause RS formation in mice. Considering the strong correlation between SF3B1 mutations and RS formation in humans, it is speculated that this discrepancy is due to minimal overlap in mis-spliced genes and events between human MDS and murine models as shown in these papers [45, 46] and/or unknown differences in mitochondrial iron metabolism between human and mouse erythroid precursors. In fact, although $A B C B 7$ is consistently downregulated in human cells with $S F 3 B 1 \mathrm{~K} 700 \mathrm{E}$ expression due to alternative splicing and subsequent NMD, this is not the case with $S f 3 b 1^{+/ \mathrm{K} 700 \mathrm{E}}$ mice because of the distinct intronic sequence in $A B C B 7$ between mouse and man. In addition, it has been reported that RSs are not observed in conditional $A b c b 7 \mathrm{KO}$ mice [59]. These results also raise another possibility that other deregulated genes, alone or in combination with $A B C B 7$ downregulation, are responsible for the formation of RS. To this end, Mupo et al. examined expression of Tmem $14 c$, Alas2, and Slc25a37 (mitochondrial iron importer) as well as $A b c b 7$ in their $S f 3 b 1^{\mathrm{K} 700 \mathrm{E} / \mathrm{WT}} \mathrm{KI}$ mice [60]. However, no altered expression of these genes was detected in LSK cells in this model [46]. With regard to SLC25A37 (also known as Mitoferrin-1), RNA-seq of MDS patient samples with or without $S F 3 B 1 \mathrm{~K} 700 \mathrm{E}$ mutations demonstrated an increase in a transcript with retained intron in $S F 3 B 1$ mutant samples compared to $S F 3 B I$ WT controls [61]. Although functional studies for this transcript have not been studied,
SLC25A37 is a protein localized in the inner membrane of the mitochondria, which acts as an importer of $\mathrm{Fe}^{2+}$ into the mitochondria in erythroid precursors, and thereby could be implicated in the pathogenesis of RS.

\section{Therapeutic intervention for SF3B1 mutant malignancies}

Compared to the remarkable progress in understanding the roles of WT and mutant SF3B1 in hematopoiesis and splicing, there has been considerably less investigation into whether cancer cells with mutant SF3BI can be pharmacologically targeted or not. According to evidence-based and consensus-based recommendations, patients with RARS or RCMD-RS with asymptomatic cytopenias and without adverse cytogenetic features do not need treatment [62]. However, aggressive therapies, including the hypomethylating agents azacitidine or decitabine and allogeneic stem cell transplantation should be considered if disease progression is observed (reviewed recently [63]).

In terms of the relationship between SF3B1 mutations and clinical response, there is an interesting observation from a phase II clinical trial of the compound luspatercept (ACE-536) for MDS patients with low or intermediate-1 International Prognostic Scoring System (IPSS) risk who are not candidates or unresponsive/refractory to erythropoiesis-stimulating agents (ESAs; ongoing study reviewed in [64]). In this study, $82 \%$ of patients achieved an erythroid hematological improvement, with a significantly higher response rate in patients with RSs and SF3B1 mutations, although the number of non-sideroblastic patients was small. Luspatercept is a fusion protein that contains a modified extracellular domain of the human activin receptor type IIB linked to a human IgG1 Fc domain, which is shown to sequester TGF- $\beta$ superfamily ligands to suppress SMAD2/3 activation [65]. It is currently unknown if the efficacy of this agent in low-risk MDS patients is due to an unrecognized association between $S F 3 B 1$ mutations and TGF- $\beta$ signaling or not. Base on the results of this phase II study, several clinical trials of luspatercept are currently ongoing for MDS patients (clinicaltrial.gov identifiers NCT02631070, NCT02268383, NCT01749514) and may clarify this association.

Hypomethylating agents are a well-established treatment option for higher risk MDS and AML and azacitidine has been shown to prolong survival in higher risk MDS patients [66]. Hypomethylating agents may also be an therapeutic option for lower risk non-del(5q) MDS patients who are unresponsive or refractory to ESAs. Intriguingly, the $S F 3 B 1$ mutation was found to be the only factor associated with a significantly improved erythroid response to azacitidine in a randomized phase II trial of azacitidine with or without 
epoetin- $\beta$ for MDS patients in low or intermediate-1 IPSS risk with transfusion dependence who showed resistance to or relapsed after high-dose ESAs [67]. According to this study, the erythroid response was $49 \%$ (29/59) in SF3B1 mutant patients versus $22 \%(6 / 22)$ in $S F 3 B 1$ WT patients $(p=0.02)$. This result may potentially indicate currently undefined links between mis-splicing and epigenetic dysregulation.

Lenalidomide is another compound approved for treating anemia in lower risk MDS patients with 5q deletion (del(5q)), where it yields transfusion independence in $60-75 \%$ of cases and a complete or partial cytogenetic response in 40-60\%. However, an analysis of clonal architecture of non-del(5q) MDS patients who were treated with lenalidomide alone or lenalidomide plus epoetin- $\beta$ showed that lenalidomide is unlikely to eradicate MDS clones bearing SF3B1 mutations [68]. The telomerase inhibitor imetelstat is another class of drug that is suggested to show preferential effects against myelofibrosis patients with $S F 3 B 1$ or $U 2 A F 1$ mutations versus patients without these mutations (complete response rate, 38 versus $4 \%, p=0.04$ ) [69]. However, testing of imetelstat in forms of MDS where $>80 \%$ of patients harbor SF3B1 mutations, including patients with RARS and RARS$\mathrm{T}$, resulted in only modest effects in these patients. For these reasons, results from larger clinical trials of imetelstat in myeloid malignancy patients are clearly needed.

Importantly, several natural products from bacterial species and their analogs have been discovered that bind SF3B1 (and possibly other components of U2 snRNP) and block early spliceosome assembly. These compounds, which include E7107 (an analog of pladienolide B) [70], spliceostatin A [71], and the sudemycins [72], are thought to inhibit the binding of the branch point binding region of U2 snRNP to the BPS and block the essential conformational change in U2 snRNP [73-76]. Obeng et al. evaluated the efficacy of E7107 against $S f 3 b 1^{+/ \mathrm{K} 700 \mathrm{E}}$ or $S f 3 b 1^{+/+}$HSPCs in a competitive transplant setting [45]. In this experiment, either CD45.2 $S f 3 b 1^{+/ \mathrm{K} 700 \mathrm{E}}$ or $S f 3 b 1^{+/+} \mathrm{BM}$ cells were transplanted into $\mathrm{CD} 45.1^{+}$recipients with $\mathrm{CD} 45.1^{+}$competitor BM cells. After measuring the $\mathrm{CD} 45.2^{+}$chimerism in the peripheral blood at 4 weeks post-transplant, the recipient mice were treated with E7107 or vehicle for a total of 10 days. Amongst these four groups, only the $S f 3 b 1^{+/ K 700 E}$ recipients treated with E7107 showed a decrease in the chimerism of CD45.2 $2^{+}$cells in both $\mathrm{BM}$ and peripheral blood at the end of treatment, indicating that $S f 3 b 1$ mutant cells are sensitive to pharmacological modulation of spliceosome compared with WT cells. Detailed analysis of chimerism in the BM revealed a statistically significant decrease in the chimerism of LK cells and a trend of decrease in that of LSK cells. Although the downstream changes in the transcriptome and protein expression induced by these drugs in SF3B1 mutant cells are unknown, these results highlighted the importance of additional studies for the evaluation of spliceosomal inhibitors in isogeneic murine MDS/AML models and, more importantly, in preclinical settings using patient-derived xenograft (PDX) models with mutant $S F 3 B 1$ expression.

We recently demonstrated that in vivo treatment with E7107 increases retention of both constitutive and alternative introns as well as cassette exon skipping, consistent with E7107 inhibiting splicing catalysis [76]. Although these effects are observed regardless of spliceosomal mutant genotype, the magnitude of splicing inhibition following E7107 treatment was more severe in myeloid leukemias with $S r s f 2$ mutation compared to $S r s f 2$ WT leukemias. This ultimately was associated with decreased disease burden in isogenic murine leukemia models with or without SRSF 2 mutations and AML PDX models. In addition, in non-competitive BM transplantation assays, recipient mice reconstituted with hemizygous $M x 1-C r e / S r s f 2^{\mathrm{P} 95 \mathrm{H} /-}$ BM mononuclear cells exhibited rapid lethality and severe BM failure due to loss of HSPCs. Conversely, the loss of HSPCs and BM aplasia were not seen in $\operatorname{Srsf} 2^{\mathrm{P} 95 \mathrm{H} /+}$ or $\operatorname{Srsf} 2^{+/-}$mice. These results, as well as the observations from in vivo E7107 treatment against $S f 3 b 1^{+/ \mathrm{K} 700 \mathrm{E}}$ hematopoietic cells, suggest that survival of spliceosomal mutant cells may depend on the activity of the residual WT allele and that cells heterozygous for spliceosomal mutations may therefore have increased sensitivity to spliceosomal inhibition (Fig. 2). An orally bioavailable analog of E7107, H3B8800 has shown promising preclinical results in isogenic SRSF2 and SF3B1-mutant leukemias [77]. These data have led to initiation of a phase I dose-escalation study of H3B8800 for patients with spliceosomal mutant MDS, AML, and CMML (clinicaltrials.gov identifier NCT02841540).

\section{Future direction}

Since the discovery of frequent mutations in genes encoding spliceosomal components in MDS and other malignancies, continuous efforts have provided deep insights into the pathogenetic functions of these spliceosomal mutations. Although promising preclinical results of spliceosomal inhibitors have been reported, it is currently unclear if these compounds are effective for cancers that bear multiple genetic defects. Therefore, it will now be important to understand the effects of spliceosomal gene mutations in the context of other genetic abnormalities commonly co-occurring with them. These include mutations in $S F 3 B 1$ and $\operatorname{inv}(3) / \mathrm{t}(3 ; 3)$, which activate the proto-oncogene ecotropic viral integration-1 (EVII) [78], as well as commonly co-existing mutations in SRSF2 and $I D H 2$ and $U 2 A F 1$ and ASXL1 [79-81]. These studies may reveal novel contributions of splicing mutations to cancer and potentially open a novel path to develop 


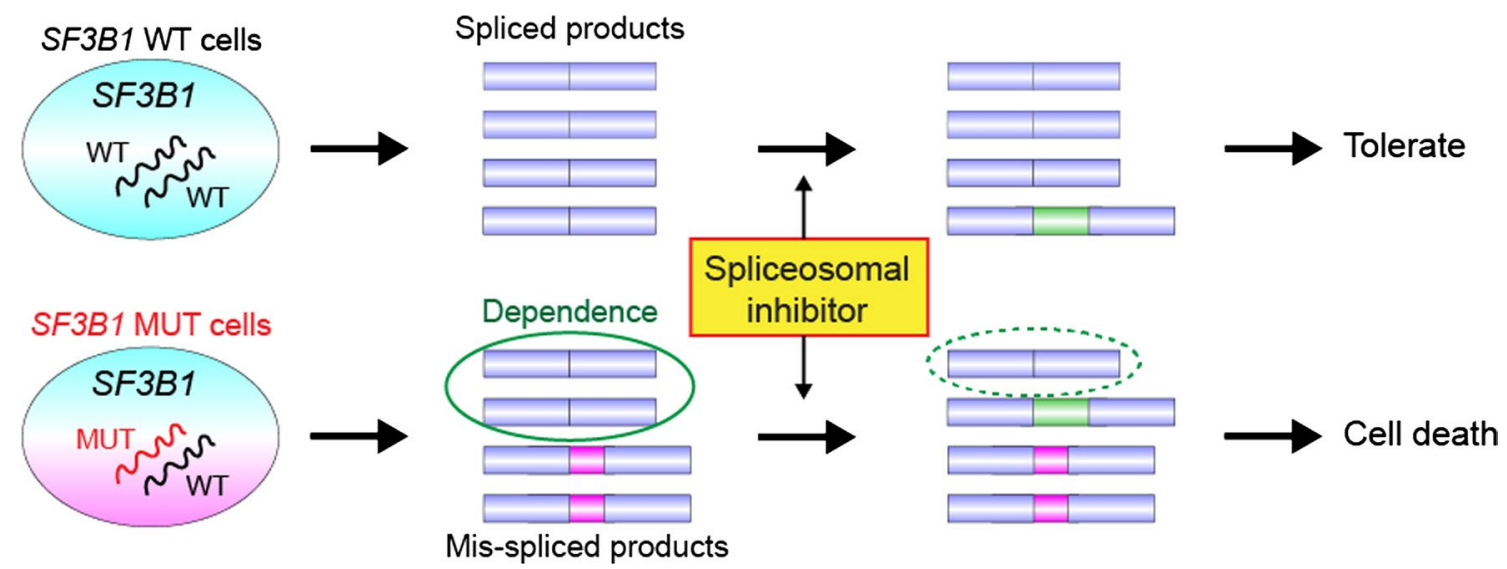

Fig. 2 A model explaining the differential effects of spliceosomal inhibition on spliceosomal mutant (MUT) cells versus wild-type (WT) cells. In this model, although spliceosomal MUT cells have increased mis-spliced products (shown as a product with additional sequence in pink) compared to WT cells, these cells can still survive depending on intact splicing products derived from a WT allele. Pharmacological inhibition of spliceosome may result in global splicing

therapeutic strategies for spliceosomal mutant cancers. Several genetically engineered spliceosomal mutant mouse models demonstrate MDS-like phenotypes, which have facilitated drug testing in vivo as described above. However, robust MDS patient-derived xenograft models suitable for preclinical studies remain elusive. Development of such models may be critically important for preclinical studies leading to curative therapies for leukemias with aberrant splicing catalysis.

\section{Compliance with ethical standards}

Conflict of interest OAW is a consultant for H3 Biomedicine Inc.

Author contributions Conception and design: AY, OAW. Writing, review, and/or revision of the manuscript: AY, OAW.

Grant support AY is supported by the Aplastic Anemia and MDS Research Foundation and Lauri Strauss Leukemia Foundation. OAW is supported by grants from the Edward P. Evans Foundation, the Department of Defense Bone Marrow Failure Research Program (BM150092 and W81XWH-12-1-0041), NIH/NHLBI (R01 HL128239), an NIH K08 Clinical Investigator Award (1K08CA160647-01), the Josie Robertson Investigator Program, a Damon Runyon Clinical Investigator Award, an award from the Starr Foundation (I8-A8-075), the Leukemia and Lymphoma Society, and the Pershing Square Sohn Cancer Research Alliance.

\section{References}

1. Arber DA, Orazi A, Hasserjian R, Thiele J, Borowitz MJ, Le Beau MM, et al. The 2016 revision to the World Health inhibition in both genotypes, typified by the widespread retention of both constitutive and alternative introns and exclusion of cassette exons characteristic of inefficient splicing catalysis (shown as a product with additional sequence in green). In contrast to WT cells that can tolerate the pharmacological inhibition of spliceosome because they still have enough intact splicing products, MUT cells no longer have enough canonical products to survive

Organization classification of myeloid neoplasms and acute leukemia. Blood. 2016;127(20):2391-405.

2. Swerdlow SH, Campo E, Harris NL, Jaffe ES, Pileri SA, Stein H, Thiele J, Vardiman JW. WHO classification of tumours of haematopoietic and lymphoid tissues, 4th edn. New York: WHO Press; 2008.

3. Germing U, Gattermann N, Aivado M, Hildebrandt B, Aul C. Two types of acquired idiopathic sideroblastic anaemia (AISA): a time-tested distinction. $\mathrm{Br} \mathrm{J}$ Haematol. 2000;108(4):724-8.

4. Papaemmanuil E, Cazzola M, Boultwood J, Malcovati L, Vyas P, Bowen D, et al. Somatic SF3B1 mutation in myelodysplasia with ring sideroblasts. N Engl J Med. 2011;365(15):1384-95.

5. Yoshida K, Sanada M, Shiraishi Y, Nowak D, Nagata Y, Yamamoto R, et al. Frequent pathway mutations of splicing machinery in myelodysplasia. Nature. 2011;478(7367):64-9.

6. Malcovati L, Karimi M, Papaemmanuil E, Ambaglio I, Jadersten $\mathrm{M}$, Jansson M, et al. SF3B1 mutation identifies a distinct subset of myelodysplastic syndrome with ring sideroblasts. Blood. 2015;126(2):233-41.

7. Malcovati L, Papaemmanuil E, Bowen DT, Boultwood J, Della Porta MG, Pascutto $\mathrm{C}$, et al. Clinical significance of SF3B1 mutations in myelodysplastic syndromes and myelodysplastic/myeloproliferative neoplasms. Blood. 2011;118(24):6239-46.

8. Cazzola M, Della Porta MG, Malcovati L. The genetic basis of myelodysplasia and its clinical relevance. Blood. 2013;122(25):4021-34.

9. Bejar R, Stevenson KE, Caughey BA, Abdel-Wahab O, Steensma DP, Galili N, et al. Validation of a prognostic model and the impact of mutations in patients with lower-risk myelodysplastic syndromes. J Clin Oncol. 2012;30(27):3376-82.

10. Patnaik MM, Lasho TL, Hodnefield JM, Knudson RA, Ketterling RP, Garcia-Manero G, et al. SF3B1 mutations are prevalent in myelodysplastic syndromes with ring sideroblasts but do not hold independent prognostic value. Blood. 2012;119(2):569-72.

11. Broseus J, Alpermann T, Wulfert M, Florensa Brichs L, Jeromin S, Lippert E, et al. Age, JAK2(V617F) and SF3B1 mutations are the main predicting factors for survival in refractory anaemia 
with ring sideroblasts and marked thrombocytosis. Leukemia. 2013;27(9):1826-31.

12. Jeromin $\mathrm{S}$, Haferlach T, Grossmann V, Alpermann T, Kowarsch A, Haferlach C, et al. High frequencies of SF3B1 and JAK2 mutations in refractory anemia with ring sideroblasts associated with marked thrombocytosis strengthen the assignment to the category of myelodysplastic/myeloproliferative neoplasms. Haematologica. 2013;98(2):e15-7.

13. Patnaik MM, Lasho TL, Finke CM, Hanson CA, King RL, Ketterling RP, et al. Predictors of survival in refractory anemia with ring sideroblasts and thrombocytosis (RARS-T) and the role of next-generation sequencing. Am J Hematol. 2016;91(5):492-8.

14. Nangalia J, Massie CE, Baxter EJ, Nice FL, Gundem G, Wedge DC, et al. Somatic CALR mutations in myeloproliferative neoplasms with nonmutated JAK2. N Engl J Med. 2013;369(25):2391-405.

15. Klampfl T, Gisslinger H, Harutyunyan AS, Nivarthi H, Rumi E, Milosevic JD, et al. Somatic mutations of calreticulin in myeloproliferative neoplasms. N Engl J Med. 2013;369(25):2379-90.

16. Broseus J, Lippert E, Harutyunyan AS, Jeromin S, Zipperer E, Florensa $\mathrm{L}$, et al. Low rate of calreticulin mutations in refractory anaemia with ring sideroblasts and marked thrombocytosis. Leukemia. 2014;28(6):1374-6.

17. Broseus J, Florensa L, Zipperer E, Schnittger S, Malcovati L, Richebourg S, et al. Clinical features and course of refractory anemia with ring sideroblasts associated with marked thrombocytosis. Haematologica. 2012;97(7):1036-41.

18. Bottomley SS, Fleming MD. Sideroblastic anemia: diagnosis and management. Hematol Oncol Clin N Am. 2014;28(4):65370 (v).

19. Fujiwara T, Harigae H. Pathophysiology and genetic mutations in congenital sideroblastic anemia. Pediatr Int. 2013;55(6):675-9.

20. Bergmann AK, Campagna DR, McLoughlin EM, Agarwal S, Fleming MD, Bottomley SS, et al. Systematic molecular genetic analysis of congenital sideroblastic anemia: evidence for genetic heterogeneity and identification of novel mutations. Pediatr Blood Cancer. 2010;54(2):273-8.

21. Cotter PD, Baumann M, Bishop DF. Enzymatic defect in "X-linked" sideroblastic anemia: molecular evidence for erythroid delta-aminolevulinate synthase deficiency. Proc Natl Acad Sci USA. 1992;89(9):4028-32.

22. Harigae H, Suwabe N, Weinstock PH, Nagai M, Fujita H, Yamamoto $\mathrm{M}$, et al. Deficient heme and globin synthesis in embryonic stem cells lacking the erythroid-specific delta-aminolevulinate synthase gene. Blood. 1998;91(3):798-805.

23. Guernsey DL, Jiang H, Campagna DR, Evans SC, Ferguson M, Kellogg MD, et al. Mutations in mitochondrial carrier family gene SLC25A38 cause nonsyndromic autosomal recessive congenital sideroblastic anemia. Nat Genet. 2009;41(6):651-3.

24. Kannengiesser C, Sanchez M, Sweeney M, Hetet G, Kerr B, Moran E, et al. Missense SLC25A38 variations play an important role in autosomal recessive inherited sideroblastic anemia. Haematologica. 2011;96(6):808-13.

25. Yien YY, Robledo RF, Schultz IJ, Takahashi-Makise N, Gwynn B, Bauer DE, et al. TMEM14C is required for erythroid mitochondrial heme metabolism. J Clin Invest. 2014;124(10):4294-304.

26. Pagon RA, Bird TD, Detter JC, Pierce I. Hereditary sideroblastic anaemia and ataxia: an X linked recessive disorder. J Med Genet. 1985;22(4):267-73.

27. D'Hooghe M, Selleslag D, Mortier G, Van Coster R, Vermeersch $\mathrm{P}$, Billiet $\mathrm{J}$, et al. X-linked sideroblastic anemia and ataxia: a new family with identification of a fourth $\mathrm{ABCB} 7$ gene mutation. Eur J Paediatr Neurol. 2012;16(6):730-5.

28. Bekri S, Kispal G, Lange H, Fitzsimons E, Tolmie J, Lill R, et al. Human $\mathrm{ABC} 7$ transporter: gene structure and mutation causing X-linked sideroblastic anemia with ataxia with disruption of cytosolic iron-sulfur protein maturation. Blood. 2000;96(9):3256-64.

29. Kispal G, Csere P, Prohl C, Lill R. The mitochondrial proteins Atm $1 \mathrm{p}$ and Nfs1p are essential for biogenesis of cytosolic $\mathrm{Fe} / \mathrm{S}$ proteins. EMBO J. 1999;18(14):3981-9.

30. Camaschella C, Campanella A, De Falco L, Boschetto L, Merlini $\mathrm{R}$, Silvestri L, et al. The human counterpart of zebrafish shiraz shows sideroblastic-like microcytic anemia and iron overload. Blood. 2007;110(4):1353-8.

31. Bykhovskaya Y, Casas K, Mengesha E, Inbal A, Fischel-Ghodsian N. Missense mutation in pseudouridine synthase 1 (PUS1) causes mitochondrial myopathy and sideroblastic anemia (MLASA). Am J Hum Genet. 2004;74(6):1303-8.

32. Riley LG, Cooper S, Hickey P, Rudinger-Thirion J, McKenzie $\mathrm{M}$, Compton A, et al. Mutation of the mitochondrial tyrosyltRNA synthetase gene, YARS2, causes myopathy, lactic acidosis, and sideroblastic anemia-MLASA syndrome. Am J Hum Genet. 2010;87(1):52-9.

33. Rotig A, Cormier V, Blanche S, Bonnefont JP, Ledeist F, Romero N, et al. Pearson's marrow-pancreas syndrome. A multisystem mitochondrial disorder in infancy. J Clin Invest. 1990;86(5):1601-8.

34. Lichtenstein DA, Crispin AW, Sendamarai AK, Campagna DR, Schmitz-Abe K, Sousa CM, et al. A recurring mutation in the respiratory complex 1 protein NDUFB11 is responsible for a novel form of X-linked sideroblastic anemia. Blood. 2016;128(15):1913-7.

35. Schmitz-Abe K, Ciesielski SJ, Schmidt PJ, Campagna DR, Rahimov F, Schilke BA, et al. Congenital sideroblastic anemia due to mutations in the mitochondrial HSP70 homologue HSPA9. Blood. 2015;126(25):2734-8.

36. Chakraborty PK, Schmitz-Abe K, Kennedy EK, Mamady H, Naas T, Durie D, et al. Mutations in TRNT1 cause congenital sideroblastic anemia with immunodeficiency, fevers, and developmental delay (SIFD). Blood. 2014;124(18):2867-71.

37. del Rey M, Benito R, Fontanillo C, Campos-Laborie FJ, Janusz $\mathrm{K}$, Velasco-Hernandez T, et al. Deregulation of genes related to iron and mitochondrial metabolism in refractory anemia with ring sideroblasts. PLoS One. 2015;10(5):e0126555.

38. Daguenet E, Dujardin G, Valcarcel J. The pathogenicity of splicing defects: mechanistic insights into pre-mRNA processing inform novel therapeutic approaches. EMBO Rep. 2015;16(12):1640-55.

39. Lee Y, Rio DC. Mechanisms and regulation of alternative premRNA splicing. Annu Rev Biochem. 2015;84:291-323.

40. Nguyen TH, Galej WP, Fica SM, Lin PC, Newman AJ, Nagai K. CryoEM structures of two spliceosomal complexes: starter and dessert at the spliceosome feast. Curr Opin Struct Biol. 2016;36:48-57.

41. Papasaikas P, Valcarcel J. The spliceosome: the ultimate RNA chaperone and sculptor. Trends Biochem Sci. 2016;41(1):33-45.

42. Yoshimi A, Abdel-Wahab O. Molecular pathways: understanding and targeting mutant spliceosomal proteins. Clin Cancer Res. 2017;23(2):336-41

43. Sahebi M, Hanafi MM, van Wijnen AJ, Azizi P, Abiri R, Ashkani $\mathrm{S}$, et al. Towards understanding pre-mRNA splicing mechanisms and the role of SR proteins. Gene. 2016;587(2):107-19.

44. Dvinge H, Kim E, Abdel-Wahab O, Bradley RK. RNA splicing factors as oncoproteins and tumour suppressors. Nat Rev Cancer. 2016;16(7):413-30.

45. Obeng EA, Chappell RJ, Seiler M, Chen MC, Campagna DR, Schmidt PJ, et al. Physiologic expression of Sf3b1(K700E) causes impaired erythropoiesis, aberrant splicing, and sensitivity to therapeutic spliceosome modulation. Cancer Cell. 2016;30(3):404-17. 
46. Mupo A, Seiler M, Sathiaseelan V, Pance A, Yang Y, Agrawal AA, Iorio F, Bautista R, Pacharne S, Tzelepis K, Manes N, Wright P, Papaemmanuil E, Kent DG, Campbell PC, Buonamici S, Bolli N, Vassiliou GS. Hemopoietic-specific Sf3b1K700E knock-in mice display the splicing defect seen in human MDS but develop anemia without ring sideroblasts. Leukemia. 2017;31(3):720-727. doi:10.1038/leu.2016.251

47. Bejar R, Stevenson K, Abdel-Wahab O, Galili N, Nilsson B, Garcia-Manero G, et al. Clinical effect of point mutations in myelodysplastic syndromes. N Engl J Med. 2011;364(26):2496-506.

48. Langemeijer SM, Kuiper RP, Berends M, Knops R, Aslanyan MG, Massop M, et al. Acquired mutations in TET2 are common in myelodysplastic syndromes. Nat Genet. 2009;41(7):838-42.

49. Darman RB, Seiler M, Agrawal AA, Lim KH, Peng S, Aird D, et al. Cancer-associated SF3B1 hotspot mutations induce cryptic $3^{\prime}$ splice site selection through use of a different branch point. Cell Rep. 2015;13(5):1033-45.

50. DeBoever C, Ghia EM, Shepard PJ, Rassenti L, Barrett CL, Jepsen K, et al. Transcriptome sequencing reveals potential mechanism of cryptic $3^{\prime}$ splice site selection in SF3B1-mutated cancers. PLoS Comput Biol. 2015;11(3):e1004105.

51. Carrocci TJ, Zoerner DM, Paulson JC, Hoskins AA. SF3b1 mutations associated with myelodysplastic syndromes alter the fidelity of branchsite selection in yeast. Nucleic Acids Res. 2017. doi:10.1093/nar/gkw1349

52. Tang Q, Rodriguez-Santiago S, Wang J, Pu J, Yuste A, Gupta $\mathrm{V}$, et al. SF3B1/Hsh155 HEAT motif mutations affect interaction with the spliceosomal ATPase Prp5, resulting in altered branch site selectivity in pre-mRNA splicing. Genes Dev. 2016;30(24):2710-23.

53. Cretu C, Schmitzova J, Ponce-Salvatierra A, Dybkov O, De Laurentiis EI, Sharma K, et al. Molecular architecture of SF3b and structural consequences of its cancer-related mutations. Mol Cell. 2016;64(2):307-19.

54. Matsunawa M, Yamamoto R, Sanada M, Sato-Otsubo A, Shiozawa Y, Yoshida K, et al. Haploinsufficiency of Sf3b1 leads to compromised stem cell function but not to myelodysplasia. Leukemia. 2014;28(9):1844-50.

55. Wang C, Sashida G, Saraya A, Ishiga R, Koide S, Oshima M, et al. Depletion of Sf3b1 impairs proliferative capacity of hematopoietic stem cells but is not sufficient to induce myelodysplasia. Blood. 2014;123(21):3336-43.

56. Visconte V, Rogers HJ, Singh J, Barnard J, Bupathi M, Traina F, et al. SF3B1 haploinsufficiency leads to formation of ring sideroblasts in myelodysplastic syndromes. Blood. 2012;120(16):3173-86.

57. Visconte V, Tabarroki A, Zhang L, Parker Y, Hasrouni E, Mahfouz R, et al. Splicing factor $3 \mathrm{~b}$ subunit 1 ( $\mathrm{sf} 3 \mathrm{~b} 1$ ) haploinsufficient mice display features of low risk myelodysplastic syndromes with ring sideroblasts. J Hematol Oncol. 2014;7:89.

58. Isono K, Mizutani-Koseki Y, Komori T, Schmidt-Zachmann MS, Koseki H. Mammalian polycomb-mediated repression of Hox genes requires the essential spliceosomal protein Sf3b1. Genes Dev. 2005;19(5):536-41.

59. Pondarre C, Campagna DR, Antiochos B, Sikorski L, Mulhern $\mathrm{H}$, Fleming MD. Abcb7, the gene responsible for X-linked sideroblastic anemia with ataxia, is essential for hematopoiesis. Blood. 2007;109(8):3567-9.

60. Dolatshad H, Pellagatti A, Fernandez-Mercado M, Yip BH, Malcovati L, Attwood M, et al. Disruption of SF3B1 results in deregulated expression and splicing of key genes and pathways in myelodysplastic syndrome hematopoietic stem and progenitor cells. Leukemia. 2015;29(8):1798.

61. Visconte V, Avishai N, Mahfouz R, Tabarroki A, Cowen J, Sharghi-Moshtaghin R, et al. Distinct iron architecture in SF3B1-mutant myelodysplastic syndrome patients is linked to an SLC25A37 splice variant with a retained intron. Leukemia. 2015;29(1):188-95.

62. Malcovati L, Hellstrom-Lindberg E, Bowen D, Ades L, Cermak J, Del Canizo C, et al. Diagnosis and treatment of primary myelodysplastic syndromes in adults: recommendations from the European LeukemiaNet. Blood. 2013;122(17):2943-64.

63. Almeida A, Fenaux P, List AF, Raza A, Platzbecker U, Santini $\mathrm{V}$. Recent advances in the treatment of lower-risk non-del(5q) myelodysplastic syndromes (MDS). Leuk Res. 2017;52:50-7.

64. Mies A, Hermine O, Platzbecker U. Activin receptor II ligand traps and their therapeutic potential in myelodysplastic syndromes with ring sideroblasts. Curr Hematol Malig Rep. 2016;11(6):416-24.

65. Suragani RN, Cadena SM, Cawley SM, Sako D, Mitchell D, Li $\mathrm{R}$, et al. Transforming growth factor-beta superfamily ligand trap ACE-536 corrects anemia by promoting late-stage erythropoiesis. Nat Med. 2014;20(4):408-14.

66. Fenaux P, Mufti GJ, Hellstrom-Lindberg E, Santini V, Gattermann N, Germing U, et al. Azacitidine prolongs overall survival compared with conventional care regimens in elderly patients with low bone marrow blast count acute myeloid leukemia. J Clin Oncol. 2010;28(4):562-9.

67. Thepot S, Ben Abdelali R, Chevret S, Renneville A, BeyneRauzy O, Prebet T, et al. A randomized phase II trial of azacitidine $+/$ - epoetin-beta in lower-risk myelodysplastic syndromes resistant to erythropoietic stimulating agents. Haematologica. 2016;101(8):918-25.

68. Chesnais V, Renneville A, Toma A, Lambert J, Passet M, Dumont $\mathrm{F}$, et al. Effect of lenalidomide treatment on clonal architecture of myelodysplastic syndromes without $5 \mathrm{q}$ deletion. Blood. 2016;127(6):749-60.

69. Tefferi A, Lasho TL, Begna KH, Patnaik MM, Zblewski DL, Finke CM, et al. A pilot study of the telomerase inhibitor imetelstat for myelofibrosis. N Engl J Med. 2015;373(10):908-19.

70. Kotake Y, Sagane K, Owa T, Mimori-Kiyosue Y, Shimizu $\mathrm{H}$, Uesugi M, et al. Splicing factor SF3b as a target of the antitumor natural product pladienolide. Nat Chem Biol. 2007;3(9):570-5.

71. Kaida D, Motoyoshi H, Tashiro E, Nojima T, Hagiwara M, Ishigami K, et al. Spliceostatin A targets SF3b and inhibits both splicing and nuclear retention of pre-mRNA. Nat Chem Biol. 2007;3(9):576-83.

72. Fan L, Lagisetti C, Edwards CC, Webb TR, Potter PM. Sudemycins, novel small molecule analogues of FR901464, induce alternative gene splicing. ACS Chem Biol. 2011;6(6):582-9.

73. Bonnal S, Vigevani L, Valcarcel J. The spliceosome as a target of novel antitumour drugs. Nat Rev Drug Discov. 2012;11(11):847-59.

74. Effenberger KA, Urabe VK, Jurica MS. Modulating splicing with small molecular inhibitors of the spliceosome. Wiley Interdiscip Rev RNA. 2017;8(2). doi:10.1002/wrna.1381

75. Folco EG, Coil KE, Reed R. The anti-tumor drug E7107 reveals an essential role for SF3b in remodeling $\mathrm{U} 2$ snRNP to expose the branch point-binding region. Genes Dev. 2011;25(5):440-4.

76. Lee SC, Dvinge H, Kim E, Cho H, Micol JB, Chung YR, et al. Modulation of splicing catalysis for therapeutic targeting of leukemia with mutations in genes encoding spliceosomal proteins. Nat Med. 2016;22(6):672-8.

77. Silvia Buonamici AY, Thomas M, Seiler M, Chan B, Caleb B, Darman R, Fekkes P, Karr C, Keaney G, Klimek V, Kunii K, Lee L, Chun-Wei Lee S, Liu X, Meeske C, Mizui Y, Padron E, Park E, Pazolli E, Prajapati S, Taylor J, Wang J, Warmuth M, Yu L, Zhu P, Abdel-Wahab O, Smith P. H3B-8800, an orally bioavailable modulator of the SF3b complex, shows efficacy in spliceosome-mutant myeloid malignancies. In: 58th ASH annual meeting and exposition abstract. 
78. Groschel S, Sanders MA, Hoogenboezem R, Zeilemaker A, Havermans M, Erpelinck C, et al. Mutational spectrum of myeloid malignancies with $\operatorname{inv}(3) / t(3 ; 3)$ reveals a predominant involvement of RAS/RTK signaling pathways. Blood. 2015;125(1):133-9.

79. Haferlach T, Nagata Y, Grossmann V, Okuno Y, Bacher U, Nagae $\mathrm{G}$, et al. Landscape of genetic lesions in 944 patients with myelodysplastic syndromes. Leukemia. 2014;28(2):241-7.

80. Metzeler KH, Herold T, Rothenberg-Thurley M, Amler S, Sauerland MC, Gorlich D, et al. Spectrum and prognostic relevance of driver gene mutations in acute myeloid leukemia. Blood. 2016;128(5):686-98.

81. Papaemmanuil E, Gerstung M, Malcovati L, Tauro S, Gundem G, Van Loo P, et al. Clinical and biological implications of driver mutations in myelodysplastic syndromes. Blood. 2013;122(22):3616-27 (Quiz 99). 